\title{
Sequence Searcher: A Java tool to perform regular expression and fuzzy searches of multiple DNA and protein sequences Francesco Marass and Chris Upton*
}

\author{
Address: Biochemistry and Microbiology, University of Victoria, Victoria, BC V8W 3P6, Canada \\ Email: Francesco Marass - fmarass@uvic.ca; Chris Upton* - cupton@uvic.ca \\ * Corresponding author
}

Published: 30 January 2009

BMC Research Notes 2009, 2:14 doi:10.1 186/1756-0500-2-14

This article is available from: http://www.biomedcentral.com/I756-0500/2//4

(C) 2009 Upton et al; licensee BioMed Central Ltd.

This is an Open Access article distributed under the terms of the Creative Commons Attribution License (http://creativecommons.org/licenses/by/2.0), which permits unrestricted use, distribution, and reproduction in any medium, provided the original work is properly cited.
Received: 3 November 2008

Accepted: 30 January 2009

\begin{abstract}
Background: Many sequence-searching tools have limiting factors for their use. For example, they may be platform specific, enforce restrictive size limits and sequences to be searched, or only allow searches of one of DNA or protein.

Findings: We present an easy-to-use, fast, platform-independent tool to search for amino acid or nucleotide patterns within one or many protein or nucleic acid sequences. The user can choose to search for regular expressions or perform a fuzzy search in which a particular number of errors is accepted during matching of a sequence. Positions of mismatches in fuzzy searches are displayed graphically the user.
\end{abstract}

Conclusion: SeqS provides an improved feature set and functions as a stand-alone tool or could be integrated into other bioinformatics platforms.

\section{Background}

Searching for specific patterns in protein and DNA sequences is a common analysis performed by molecular biologists. Detection of restriction enzyme cleavage sites in DNA sequences was an early use of this pattern matching process. Later, as the protein databases grew, the PROSITE motif database was developed [1]. These protein motifs are written as regular expressions that capture the variability within a consensus sequence from a short, highly conserved region in a multiple alignment. As the volume and diversity of genomic information grows, it is necessary to modify PROSITE patterns to allow them to match more diverse homologs. Searching through genomic sequences for conserved nucleotide patterns such as transcription factor binding sites is another use for this type of analysis. Large-scale sequencing has lead to some automated bioinformatics analyses, but pattern searching is such a common "hands on" interactive proce- dure that we have developed an easy-to-use tool, Sequence Searcher (SeqS), that supports searching for user-specified patterns in multiple protein and nucleotide sequences. SeqS has been integrated into several of the Viral Bioinformatics Resource Center tools and can therefore read sequences directly out of the VOCs database [2], but it also function as a stand-alone program with the ability to manage sequences much larger than viral genomes.

\section{Algorithms}

We implemented a brute-force fuzzy search algorithm and made use of the Jakarta ORO libraries [3] for Perl-like regular expressions. To speed up the searches and reduce memory requirements, an algorithm to reverse both fuzzy patterns and regular expressions was developed. In a search on DNA, SeqS first searches the top strand (the sequence itself), and then the bottom strand. However, 
rather than creating a duplicate (the bottom strand) of the nucleotide sequence, which is expensive in terms of memory, SeqS reverses and complements the query and searches the top strand again.

\section{Implementation}

In the development of SeqS, our goal was to produce an intuitive interface with easily understood output. The stand-alone interface of SeqS is simple and consists of two panels, accessed by the input and results tabs (Figure 1). In the input panel, users can import sequences to the workspace, set the search parameters and initiate the search. SeqS accepts one or many sequences (FASTA format) as input, either imported from a text file or directly pasted into a text box (add manually). Sequence names are shown in the List of target sequences box and the program uses selected sequences, or all sequences if none are selected. Sequence Searcher recognises ambiguity codes for amino acids and nucleotides as described by IUPAC [4], and tries to determine if a sequence contains nucleic acid or amino acids. SeqS supports two search types: regular expression and fuzzy. Although a regular expression may describe variation (AAA [CG], AAA followed by C or G), these searches are always exact and in this example only match patterns specified by the user (AAAC or AAAG). Supported regular expression patterns are characters (such as A and C), character sets (such as [AG]), quantifiers (such as $+, ?^{*},\{n, m\}$ ) and back references (such as (NNNN) $\backslash 1)$. The pattern AAA $\left[{ }^{\wedge} \mathrm{C}\right][\mathrm{GT}] .\{1,10\}(\mathrm{AT}) \backslash 1$ translates to AAA, anything but $\mathrm{C}$, then $\mathrm{G}$ or $\mathrm{T}$, anything 1-10 times, AT repeated twice. A useful feature of regular expressions is the ability to allow variable space between 2 patterns. In contrast, a fuzzy

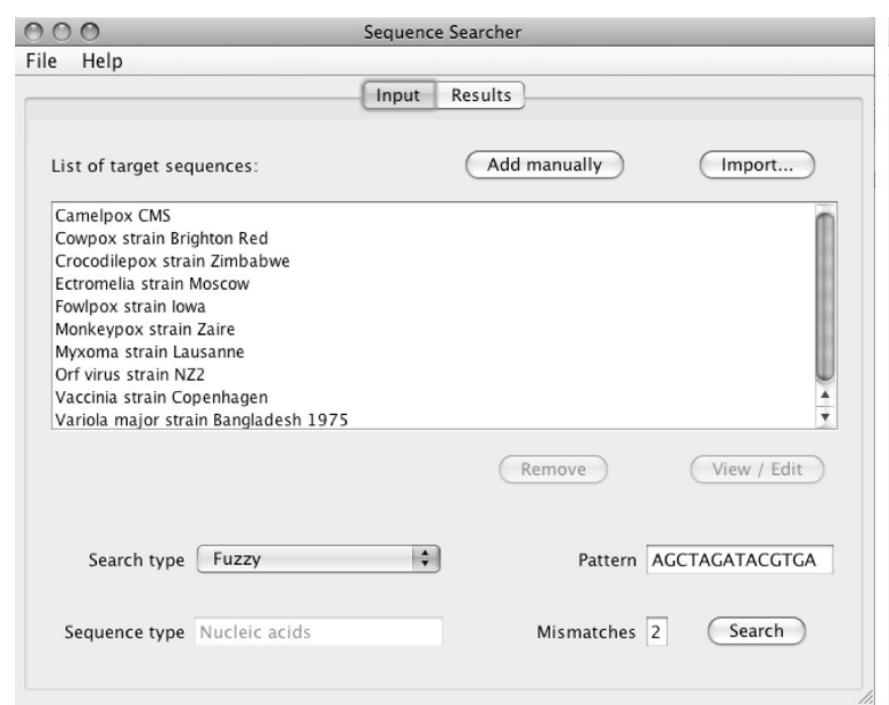

search allows a specified number of mismatches within the pattern; e.g. ATGATAGT with 3 mismatches. However, the fuzzy search also supports a basic syntax similar to regular expressions to allow even more flexible pattern recognition; it is possible to match one of some characters by listing them within square brackets ([AG] matches either A or $G$ ) and it is also possible to exclude characters by placing them within braces $(\{A G\}$ matches neither A nor $\mathrm{G})$. SeqS allows the number of mismatches to be $0-40 \%$ of the pattern length; this prevents the search from taking too long and returning too many imprecise matches.

Upon completion of a search, the results panel is presented, showing the data in tabular format (Figure 1). It is possible to sort the table by any data column using the column header (sequence, match, match start, match stop, confidence, strand) as well as filter the results set by sequence and by strand using drop-down menus. The search parameters are also reported. To facilitate interpretation of fuzzy search results, an additional graphical representation of the match is included in the form of a multi-coloured line that is divided into a series of segments numbering equal to the length of the pattern match. A segment is coloured green if a character match is exact, orange if it matches an ambiguity character and red in the case of a mismatch. The user can choose to save the results (all or selected rows) to a tab-delimited text file that is easily imported into a spreadsheet for further analysis.

SeqS is limited by the amount of memory dedicated to the Java Virtual Machine and may run out of memory during

\section{Figure I}

SeqS user interface. Left side: Input panel, 10 poxvirus genomes searched with a fuzzy pattern; right side: Results panel,

showing positions of mismatches within matched pattern.

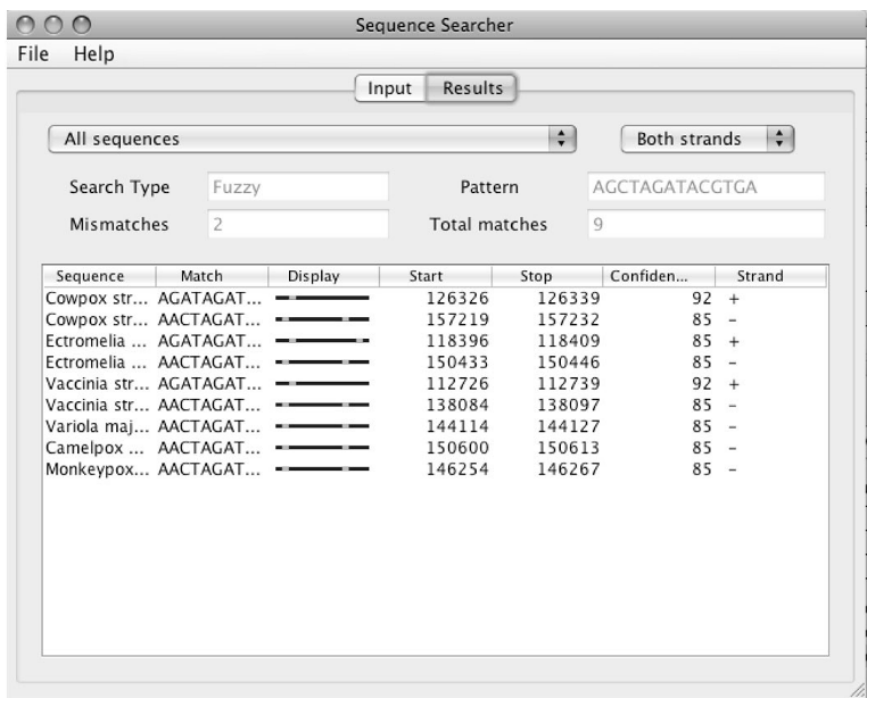


Table I: Examples of SeqS searches on DNA sequences totalling 170 MB. All searches took less than 4 seconds

\begin{tabular}{lcccc}
\hline & Fuzzy Search & & Regular Expression Search \\
\hline Search Pattern & No. of mismatches & No. of hits & Search Pattern & No. of hits \\
\hline ACGTACGTA & 0 & 44 & ACGTACGT & 216 \\
\hline ACGTACGTA & 1 & 1808 & ACGACGTA & 108 \\
\hline ACGTACGTA & 2 & 22124 & ACGACGT & ACGA [CGTA]GT \\
\hline ACGTACGTACGT & 1 & 24 & ACGA.G\{I,3\}T & 1272 \\
\hline ACGTACGTACGT & 2 & 672 & ACGA.\{I,50\}G $\{1,3\} T$ & 23991 \\
\hline ACGTACGTACGTACGT & 3 & 80 & &
\end{tabular}

import of sequences or performing a search. However, for most practical work with viral and bacterial genomes, these limits are of no consequence. For example, Table 1 shows a series of searches performed with a set of sequences totalling $170 \mathrm{Mb}$ that each took $4 \mathrm{~s}$ or less (iMac with $2.4 \mathrm{GHz}$ Core 2 Duo and 4 GB RAM). SeqS imports the test sequences at approximately $1 \mathrm{Mb} / \mathrm{s}$.

\section{Conclusion}

SeqS is a versatile tool that can be used as a stand-alone program or easily incorporated into more complex bioinformatics workbenches. It provides the ability to search multiple sequences in a single run with regular expressions or fuzzy patterns. Results are displayed in sortable tables and graphics are used to show fuzzy matches. To enable viewing of results with genome annotations, the core of SeqS has been incorporated in to the Viral Genome Organizer [5] and Base-By-Base [6] tools that can read GenBank files.

\section{Availability and requirements}

Project name: Sequence Searcher (SeqS)

Project homepage: http://www.virology.ca/tools/ SequenceSearcher

Operating system: Platform independent

Programming language: Java

Other requirements: Java 1.4 or higher; Java Web Start

License: SeqS is distributed under the Open Software License.

Any restrictions to use by non-academics: None

\section{Competing interests}

The authors declare that they have no competing interests.

\section{Authors' contributions}

CU conceived the idea and specifications; FM developed the code; both authors tested the tool and contributed to writing the manuscript.

\section{Acknowledgements}

This work was supported by NIAID grant HHSN266200400036C.

\section{References}

I. Sigrist C], Cerutti L, Hulo N, Gattiker A, Falquet L, Pagni M, Bairoch $A$, Bucher P: PROSITE: a documented database using patterns and profiles as motif descriptors. Brief Bioinform 2002, 3(3):265-274.

2. Ehlers A, Osborne J, Slack S, Roper RL, Upton C: Poxvirus Orthologous Clusters (POCs). Bioinformatics 2002, I 8(I I): 1 544- 1545.

3. Apache Software Foundation, Jakarta ORO [http:// jakarta.apache.org/oro/index.html]

4. Nomenclature for Incompletely Specified Bases in Nucleic Acid Sequences [http://www.chem.qmul.ac.uk/iubmb/miscl naseq.html]

5. Upton C, Hogg D, Perrin D, Boone M, Harris NL: Viral genome organizer: a system for analyzing complete viral genomes. Virus Res 2000, 70(I-2):55-64.

6. Brodie R, Smith AJ, Roper RL, Tcherepanov V, Upton C: Base-ByBase: single nucleotide-level analysis of whole viral genome alignments. BMC Bioinformatics 2004, 5:96. 